\title{
About the Regularities of Fatigue Fracture and Fatigue Strength Due to the Influence of Asymmetry of the Tension Cycle
}

\author{
V. P. BAGMUTOV*, A. A. BELOV**, V. I. VODOPYANOV***, O. V. KONDRATYEV**** \\ *Volgograd State Technical University, pr. Lenina 28, 400005 Volgograd, Russian Federation, E-mail: sopromat@vstu.ru \\ **Volgograd State Technical University, pr. Lenina 28, 400005 Volgograd, Russian Federation, \\ E-mail: belovaa58@gmail.com \\ ***Volgograd State Technical University, pr. Lenina 28, 400005 Volgograd, Russian Federation, \\ E-mail: vivodopynov@mail.ru \\ ****Volgograd State Technical University, pr. Lenina 28, 400005 Volgograd, Russian Federation, \\ E-mail: olekond@yandex.ru
}

cross $^{\text {ref }}$ http://dx.doi.org/10.5755/j01.mech.24.5.14474

\section{Introduction}

An experiment is a main source of fatigue properties evaluation. Existing approaches of experimental research under cyclic loads are associated with a high labor intensity testing, long periods of their implementation, making it difficult for evaluation of fatigue characteristics, in particular, new materials or modifications thereof. Known calculation methods do not provide the necessary accuracy because of a lack of information about the specific properties due to the operating conditions [1]. One of the possible solutions for this problem is the use of experimental and computational models, which have been tested to the actual working conditions of the product [2]. Destruction of materials under cyclic loading occurs in the tension range between a temporary resistance $\sigma_{u}$ and limit of endurance $\sigma_{R}[1-4]$. The experimental results are presented in the form of three stages of the fatigue curve: quasi-static, transient and pure fatigue [3]. One of the issues that are of practical interest is determination regularities of influence asymmetry coefficient of stress cycle ( $R_{\sigma}=\sigma_{\min } / \sigma_{\max }$ ) on the curve of fatigue [1]. The purpose of this work is to research the influence of asymmetry of the stress cycle on the fatigue fracture regularities, detected by a change in the parameters of the fatigue curve in the transition stage from quasi-static to fatigue fracture.

\section{Experimental details}

The tests were conducted on samples of steel 45 (GOST 1050-2013), and the titanium alloy PT-3V (GOST 19807-91. The choice of materials is dictated by their characteristic properties: at approximately the same strength characteristics, and the relation yield stress and ultimate stress for steel 45 is 0.6 , and for PT-3V - 0.9 or more. The index of deformed hardening $\mathrm{n}$ in the expression $\sigma=\sigma_{0} \varepsilon^{n}$ is 0.06 , and $0.16-0.18$ for titanium alloy and steel 45, respectively [GOST 25.503-97].

For fatigue tests produced specimens, the outline of which is shown in Fig. 1

The chemical composition and mechanical characteristics of the tested materials are presented in Table 1 and Table 2.

Steel specimens fabricated from rod, titanium specimens carve out from sheet. The cutting direction of the sheet coincided with the rolling direction. The minimum diameter of specimen is $8 \mathrm{~mm}$.

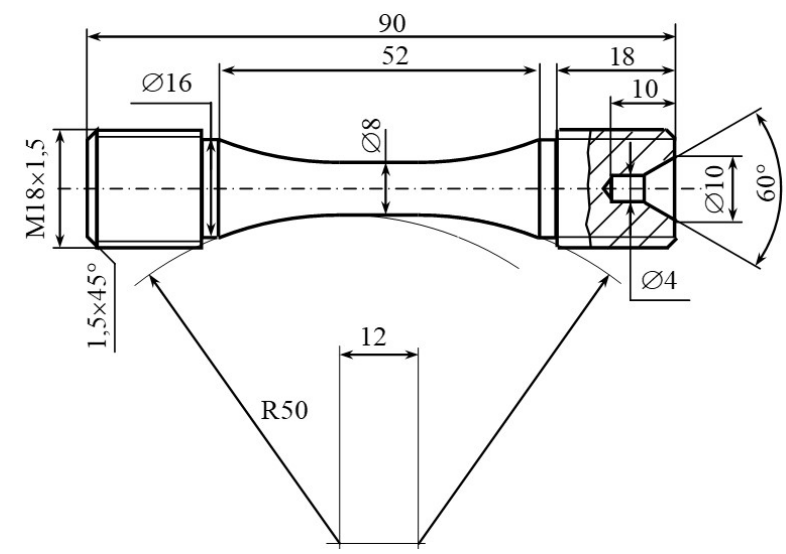

Fig. 1 The specimen for the cyclic tests

Table 1

Chemical compositions of tested materials

\begin{tabular}{|l|c|c|c|c|c|}
\hline \multirow{4}{*}{$\begin{array}{l}\text { Steel } \\
45\end{array}$} & $\mathrm{C}$ & $\mathrm{Si}$ & $\mathrm{Mn}$ & $\mathrm{Cr}$ & $\mathrm{Ni}$ \\
\cline { 2 - 6 } & 0.47 & 0.28 & 0.72 & 0.25 & 0.22 \\
\cline { 2 - 6 } & $\mathrm{Cu}$ & $\mathrm{S}$ & $\mathrm{P}$ & & \\
\cline { 2 - 6 } & 0.01 & 0.015 & 0.013 & & \\
\hline \multirow{4}{*}{ PT-3V } & $\mathrm{Al}$ & $\mathrm{V}$ & $\mathrm{Fe}$ & $\mathrm{Zr}$ & $\mathrm{Si}$ \\
\cline { 2 - 6 } & 4.7 & 1.9 & 0.01 & 0.03 & 0.01 \\
\cline { 2 - 6 } & $\mathrm{C}$ & $\mathrm{N}$ & $\mathrm{H}$ & $\mathrm{O}$ & \\
\cline { 2 - 6 } & 0.06 & 0.02 & 0.003 & 0.09 & \\
\hline
\end{tabular}

Table 2

Mechanical characteristics of tested materials

\begin{tabular}{|c|c|c|c|c|}
\hline Material & $\begin{array}{c}\text { Yield } \\
\text { stress } \\
\sigma_{y}, \mathrm{MPa}\end{array}$ & $\begin{array}{c}\text { Ultimate } \\
\text { stress } \\
\sigma_{u}, \mathrm{MPa}\end{array}$ & $\begin{array}{c}\text { Relative } \\
\text { elongation } \\
\delta, \%\end{array}$ & $\begin{array}{c}\text { Reduction } \\
\text { of area } \\
\psi, \%\end{array}$ \\
\hline Steel 45 & 385 & 750 & 20 & 50 \\
\hline PT-3V & 670 & 730 & $13-15$ & $32-34$ \\
\hline
\end{tabular}

Cyclic loading by tension-compression at a frequency of 325 cycles/min is performed on a hydraulic pulsator MUP-20, modernized for cyclic tests with a negative (compressive) component of the cycle. The cyclic loading was made at the following values of the asymmetry cycle ratio: steel $45-$ at $R_{\sigma}=0.5 ; 0.25 ; 0 ;-0.25 ;-0.5 ;-1 ;-2$; 
titanium alloy - at $R_{\sigma}=0 ;-1 ;-2$. The durability of the materials was evaluated by the number of cycles before destruction of the specimens.

\section{The regularities of fatigue fracture and fatigue strength}

The dependence of the length and position of the transitions of the fatigue curves corresponding to the change of fracture mechanism from quasi-static (line $\mathrm{ab}$ ) to fatigue (line cd), on asymmetry cycle ratio $R_{\sigma}$ for steel 45 are shown in Fig. 2 in the coordinates $\sigma \sim \lg N$. The experimental results were approximated by linear dependences. The following features may be noted position change fatigue curves depending on the magnitude of the coefficient of asymmetry of the stress cycle. First, the slope of the fatigue curves depends on $R_{\sigma}$, the angle of inclination to the horizontal axis decreases at high $\left(R_{\sigma}=\right.$ $0.5)$, and low $\left(R_{\sigma}=-2\right)$ values of the asymmetry ratio. The boundary of the quasi-static fracture in durability shifts towards lower values of load cycles from $6 \cdot 10^{5}$ cycles at $R_{\sigma}=0.5$ to $5 \cdot 10^{2}$ at $R_{\sigma}=-1$, and then rises again to $10^{4}$ cycles at $R_{\sigma}=-2$. Similarly, a change in numbers of damaging of endurance cycles was detected. When $R_{\sigma}=0.5$ endurance limit is reached at $6 \cdot 10^{6}$ cycles, $R_{\sigma}=-1$ is reduced to $3 \cdot 10^{5}$ cycles, $R_{\sigma}=-2$ is increased to $2 \cdot 10^{6}$ cycles.

Similar regularities of influence $R_{\sigma}$ on the fatigue fracture stage boundaries are confirmed when testing samples of PT-3V alloy (Fig. 3).

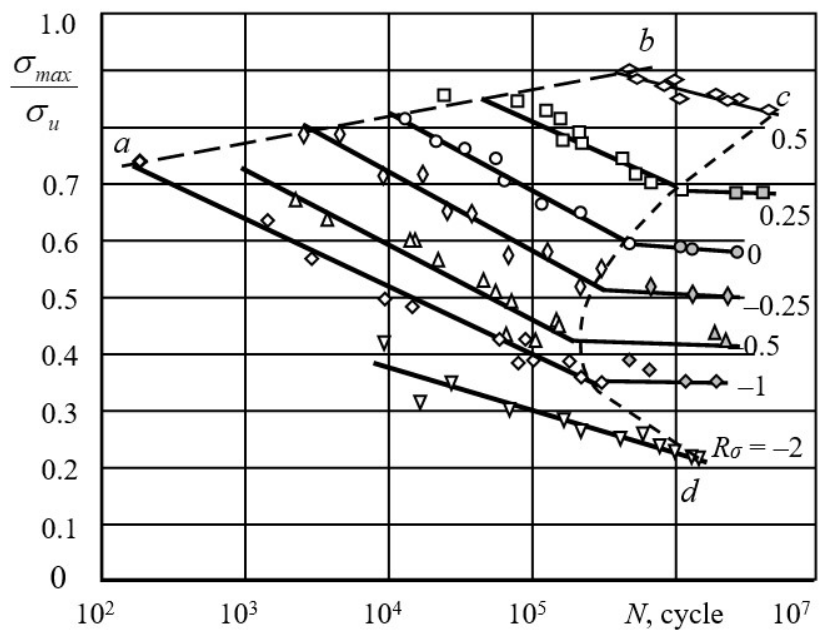

Fig. 2 Curves of the transition stage of fatigue fracture specimens of steel 45 from quasi-static destruction (ab) to fatigue failure (cd) for the coefficient of asymmetry of the stress cycle $R_{\sigma}$

As a result, there are the following regularities of influence of stress cycle asymmetry on the duration of the fatigue fracture period. In the range of variation $R_{\sigma}$ from -1 to 0.5 there is a growing number of load cycles until the stage of fatigue fracture. Beginning of the fatigue fracture stage earliest, with a smaller number of loading cycles, is observed at $R_{\sigma}=-1$ and increases by several orders of magnitude when $R_{\sigma}=0.5$. When $R_{\sigma}<-1$, the number of cycles of the beginning stage of fatigue fracture is increasing again. Although fatigue fracture stage at $R_{\sigma}=-1$ covers three orders of durable, that is, from $10^{2}$ to $10^{5}$, the total number of cycles at this stage of an order of magnitude less than at $R_{\sigma}=0.5$.

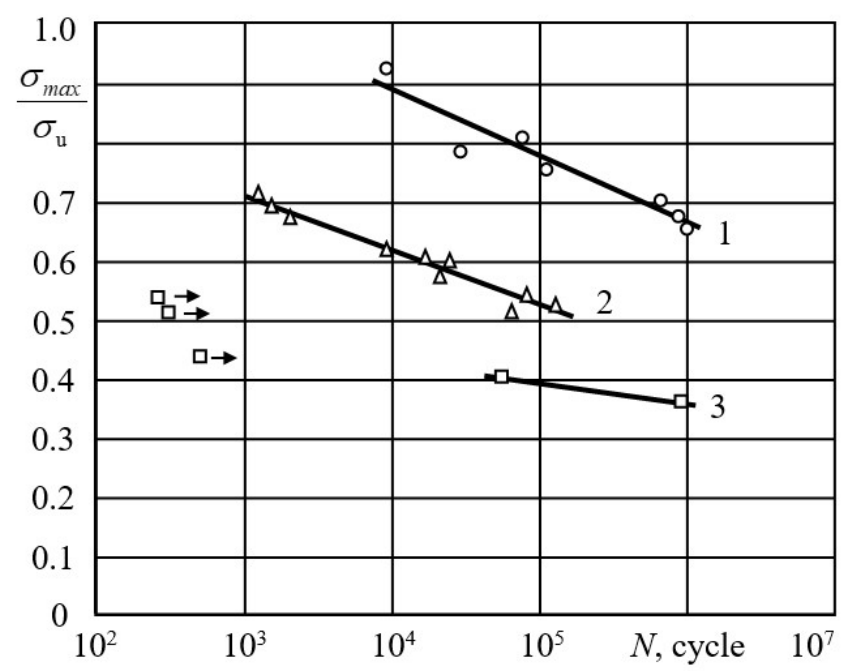

Fig. 3 Curves of the fatigue fracture specimens of the titanium alloy PT-3V for the coefficient of asymmetry of the stress cycle: $1-R_{\sigma}=0 ; 2-R_{\sigma}=-1 ; 3-R_{\sigma}=$ -2 . Points with arrows correspond to the specimens at $R_{\sigma}=-2$. Its tests have been finished owing to the accumulation of large deformations of cyclic creep

For evaluation regularities of influence $R_{\sigma}$ on the curves parameters of cyclic destruction, the experimental results were fitted in the form of:

$$
\frac{\sigma_{\max }}{\sigma_{u}}=C_{R}-K_{R} \cdot \lg N_{f},
$$

where: $\sigma_{\max } / \sigma_{u}$ - is a relative value of maximum stress of the cycle; $N_{f}$ - is a number of cycles to destruction; $C_{R}$ and the $K_{R}$ - ratios. $C_{R}$ is coefficient corresponding to the stress level at which the durability of the specimen is one cycle, that is, $\lg N_{f}=0$. It characterizes the displacement of the fatigue curve along the axis of the number of cycles. $C_{R}$ characterizes the degree of reduction of fatigue strength while the growth of longevity; determined by the angle of slope of the fatigue curve in this area.

The collected data used for constructed graphs of change parameters $C_{R}$ and $K_{R}$ in accordance to $R_{\sigma}$ (Fig. 4). As can be seen, for the fatigue curves corresponding to the fatigue fracture kind, $C_{R}$ and $K_{R}$ are nonlinear functions of $R_{\sigma}$.

The change parameter $C_{R}$, in the range of $2<R_{\sigma}<0$, can be represented by a linear function of asymmetry sress cycle ratio $R_{\sigma}$, and in the interval $0<R_{\sigma}<0.5$ behavior of the $C_{R}$ ratio is more complex. $C_{R}$ varies little in the interval $0<R_{\sigma}<0.25$ and decreases sharply when $R_{\sigma}>$ 0.25 .

Experimental results on $C_{R}$ change for PT-3V alloy obtained for $-2<R_{\sigma}<0$, are practically the same. These data are consistent with the initial ultimate stress of the tested materials.

Changing $K_{R}$ (Fig. 4) is more complicated. For the materials under study, the value of the $K_{R}$ parameter smoothly increases in the range of the coefficient of 
asymmetry of the stress cycle from -2 to zero, and then falls sharply. For PT-3V alloy, the dependence is similar, although the coefficient values are lower: the experimental curves are almost equidistant.

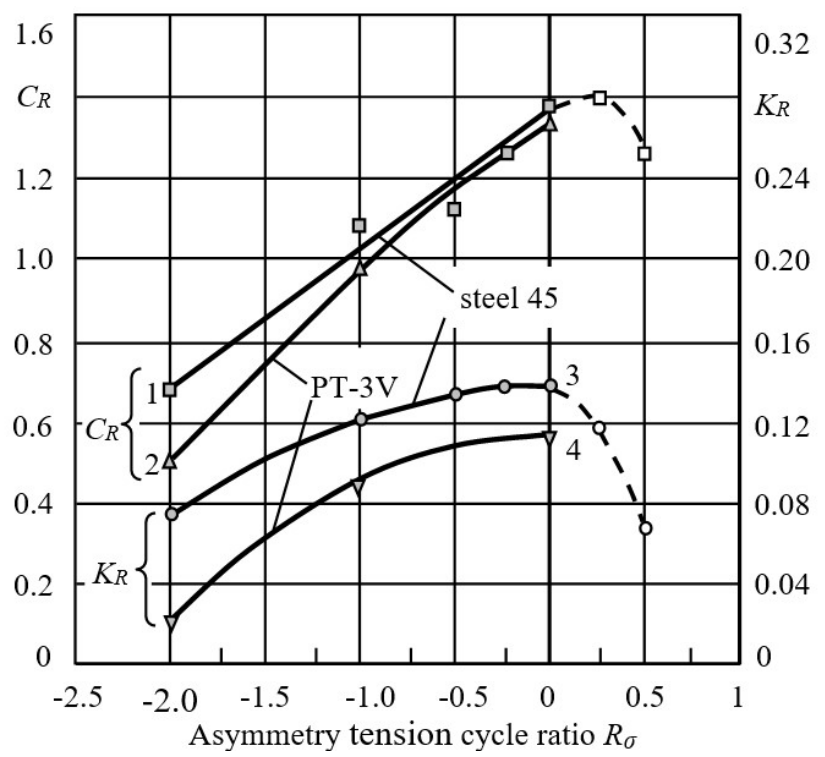

Fig. 4 Changing $C_{R}$ parameters $(1,2)$ and $K_{R}(3,4)$ in the equation $\frac{\sigma_{\max }}{\sigma_{\mathrm{B}}}=C_{R}-K_{R} \cdot \lg N_{\mathrm{p}}$ respectively, for steel 45 (1 and 3), and PT-3V alloy (2 and 4)

These results allow us to calculate the curves of fatigue fracture within the boundaries from quasi-static phase of destruction to limit of endurance. The border area, corresponding to the change from the quasi-static fracture mechanism to transition at different values $R_{\sigma}$ in semilogarithmic coordinates, can be represented by a straight line (Fig. 2, ac) [5]. The equation of this line can be used to estimate the number of cycles corresponding to the upper boundary of the fatigue fracture stage. At Fig. 2, this boundary for steel 45 is represented by a straight $a b$ and within $R_{\sigma}$ range from -1 to 0.5 is approximated by the equation:

$$
\frac{\sigma_{\max }}{\sigma_{u}}=b^{*}+a^{*} \cdot \lg N_{f u},
$$

when: $a^{*}=0.046$ and $b^{*}=0.65, N_{f u}-$ destructive number of cycles of the upper boundary of the transition stage.

From the Eqs. (1) and (2) determine:

$$
N_{f u}=\exp \frac{C_{R}-b^{*}}{a^{*}+K_{R}} .
$$

Destructive number of cycles $N_{f d}$ corresponding to the lower boundary of the transition stage of the fatigue fracture curve for a given $R_{\sigma}$ (Fig. 2, cd line), can be determined from the expression (1) with $\sigma_{\max }$ equal to the corresponding endurance limit $\sigma_{R}$. The endurance limit $\sigma_{R}$ for a given $R_{\sigma}$ is conveniently determined from the diagram of the limiting amplitudes in the coordinates $\sigma_{m} \sim$ $\sigma_{a}$. Here $\sigma_{m}=\left(\sigma_{\max }+\sigma_{\min }\right) / 2$ is the mean stress of the cycle, $\sigma_{a}=\left(\sigma_{\max }-\sigma_{\min }\right) / 2$ is the amplitude of the stress.
Limit amplitude diagram allows to predict the limit state of the material using a limited number of experimental data. According to the experimental results for steel 45 diagram of the limiting amplitudes is presented in Fig. 5, a, a for PT-3V alloy - in Fig. 5, b. The value of the endurance limit at any value of $R_{\sigma}$ can be determined in the diagram as the sum $\sigma_{\max }=\sigma_{m}+\sigma_{a}$. Thus, the parameters of the curve $c d$ (Fig. 2) allows you to determine the curve of the limiting amplitudes (Fig. 5, a). In fact, this is an estimate of the limiting states on the criterion of fatigue strength. The line corresponding to the yield strength (Fig. 5, a, curve mn) limits the stress according to the criterion of the static strength. Comparison of the position of these lines for the investigated alloys (Fig. 5, a, b) allows to reveal features of resistance to cyclic loadings depending on $R_{\sigma}$. The magnitude of safe stress amplitudes according to the fatigue criterion for PT-3V is much larger than for steel 45 . For steel 45 in the diagram of limiting amplitudes $0<R_{\sigma}<+1$, there is a substantial area of the stress in which the danger of fatigue fracture is excluded, however, there are plastic deformations, because of which the cyclic strength of steel 45 cannot be realized.
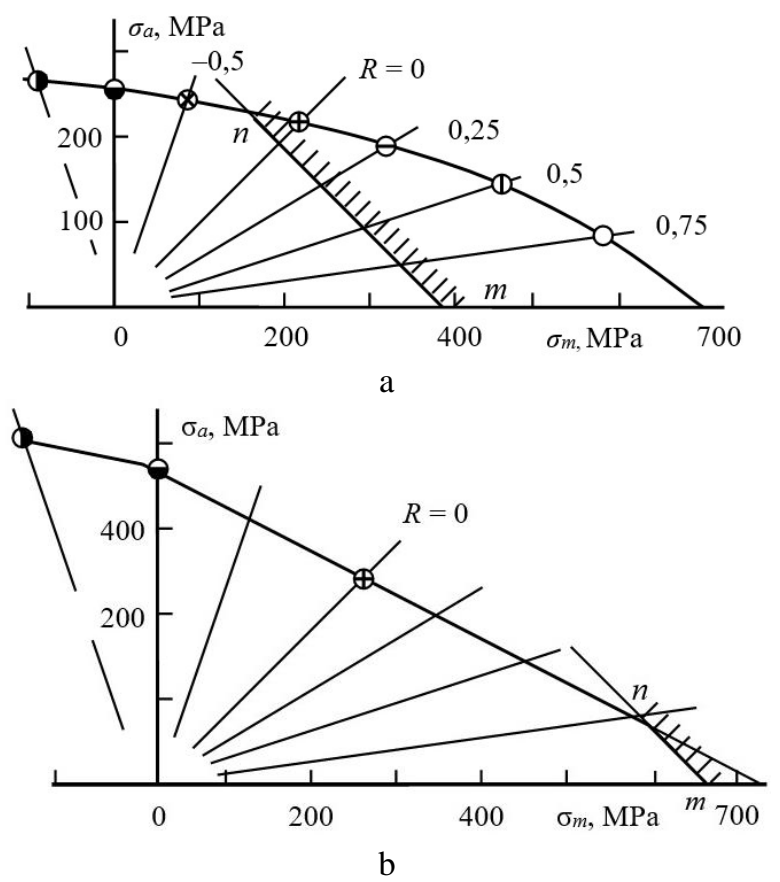

Fig. 5 Diagrams of limiting amplitudes of steel 45 (a) and PT-3V alloy (b)

This dependence of the properties of materials with approximately the same values of tensile strength can be explained by the laws of resistance to small plastic deformations. As noted above, steel 45 is characterized by a high capacity for plastic deformation. These include such indicators as the ratio of yield strength to tensile strength, the index of deformation hardening, the ratio between the tensile strength and the endurance limit. Therefore, cyclic loading with positive coefficients of cycle asymmetry allows the material to more fully realize its reserve of plasticity, increasing the area of quasi-static fracture and significantly reducing the stage length of fatigue fracture.

This circumstance determines the existence of large area $R_{\sigma}$ cycles from $R_{\sigma}=0$ to $R_{\sigma}=+1$ in limiting the amplitudes of diagram of steel 45 (Fig. 5, b), which doesn't 
have danger of fracture, but plastic deformations take place, and because of the fatigue strength cycle, which is detected in experiments with steel 45 , can not be realized.

\section{Conclusions}

1. Fatigue tests were carried out for steel 45 , titanium alloy PT-3V and built fatigue curves in transition from quasi-static to fatigue failure are constructed at different asymmetries of stress cycles $R_{\sigma}$ in the range from -2 to +0.5 . The loading was carried out by tensioncompression with the control of stresses on the loads. The transition section of the fatigue curve is approximated by linear dependencies in semi-log coordinates.

2. The influence of the stress cycle asymmetry coefficient on the length and position of the transition section of the fatigue curve in the interval from the quasistatic fracture boundary to the fatigue fracture boundary is investigated. With the decrease of from +1 to -1 the intensity of fatigue damages increases. Most intensively these processes are observed at $R_{\sigma}=-1$ and accompanied by a shift in the fatigue curve in the direction of reducing the endurance limit and longevity.

3. Based on test results built diagrams of limiting amplitudes. For steel 45, the diagram has a convex appearance, and for titanium alloy PT-3B - linear. For steel 45 in the range of changes of the coefficient of asymmetry of the stress cycle $\left(0<R_{\sigma}<1\right)$ limit state is limited to quasi-static mechanism, while, as for the alloy PT-3V quasi-static fracture occurs when $R_{\sigma}>0.75$. The observed difference in the properties of the alloys under study is associated with different resistance to small plastic deformations of the alloys under study

4. A method for calculating the position and longness of the transition part of the fatigue curve depending on the stress cycle asymmetry coefficient is proposed. The values of parameters are found, which allow to calculate the transition part of the fatigue curve at $-1<R_{\sigma}<0.5$ and to determine the coordinates of the boundaries of quasistatic and fatigue destruction types. The obtained results allow to reduce the number of labor-intensive tests necessary for the construction of fatigue curves of the transient phase at different values of the stress cycle asymmetry coefficient.

\section{Acknowledgements}

This work was supported by the Russian Foundation for Basic Research (project nos. 17-08-01742_a).

\section{References}

1. Troshchenko, V. T. 2000. The mechanical behavior of materials under different types of loading, Kiev: Logos $571 \mathrm{p}$.

2. Bagmutov, V. P.; Kondrat'ev, O. V. 2001. About resource of plasticity in asymmetrical cyclic loading of metal samples, Nauki Techniczne 138: 33-40.

3. Terentev, V. F. 2002. Fatigue strength of metals and alloys, Moscow: Intermet Engineering. 288 p.

4. Ilyin, A. A.; Kolachev, B. A.; Polkin, I. S. 2009. Titanium alloys. The composition, structure and properties. Moscow: VILS-MATI. 520 p.

5. Troshchenko, V. T.; Strizhalo, V. A.; Sinyavsky, D. P.; Ivakhnenko, V. V. 1982. The influence effect of the tension asymmetry cycle for the process of quasistatic and fatigue destruction under low-cycle loading, Problems of strength 3: 14-21.

V. P. Bagmutov, A. A. Belov, V. I. Vodopyanov, O. V. Kondratyev

ABOUT THE REGULARITIES OF FATIGUE FRACTURE AND FATIGUE STRENGTH DUE TO THE INFLUENCE ASS YMMETRY OF THE TENSION CYCLE

S u m m a r y

The fatigue curves are obtained and the regularities of the effect of the tensile cycle asymmetry in the range $-2 \leq R \leq 0.5$ on the length and position of the transition section of the fatigue curve of steel 45 and titanium alloy PT-3V are investigated. Diagrams of limit amplitudes for the studied alloys are constructed. A method for calculating the position and length of the transition stage of the fatigue curve is proposed.

Keywords: fatigue fracture, the asymmetry cycle ratio, 45 steel, titanium alloy PT-3V, the curve of fatigue.

Received March 28, 2016 Accepted October 18, 2018 